\title{
Callias' Index Theorem, Elliptic Boundary Conditions, and Cutting and Gluing
}

\author{
Johan Råde \\ Department of Mathematics, Stanford University, Stanford, CA 94305, USA \\ Email: rade@math stanford.edu
}

Received: 5 February 1993/in revised form: 15 June 1993

\begin{abstract}
It is shown that elliptic boundary conditions play the same role in Callias' index theorem as spectral boundary conditions do in the Atiyah-PatodiSinger index theorem. This is used to generalize Callias' index theorem to arbitrary complete spin-manifolds.
\end{abstract}

\section{The Index Formula}

Let $X$ be a complete odd-dimensional smooth oriented spin-manifold, with complex spinor bundle $S$. Let $V$ be a smooth Hermitian vector bundle over $X$, with a smooth unitary connection $A$ and a smooth Hermitian endomorphism $\Phi$. Let $\not_{A}$ denote the coupled Dirac operator acting on sections of $S \otimes V$. Form the operators

and

$$
D=\not \not_{A}+i 1 \otimes \Phi
$$

$$
D^{*}=\not_{A}-i 1 \otimes \Phi
$$

acting on sections of $S \otimes V$.

The index problem for such operators was first studied by C. Callias [C], who proved an index theorem in the case $X=\mathbb{R}^{2 n+1}$, using traces of integral kernels. Callias' index theorem can also be derived from Fedosov's index theorem for elliptic operators on Euclidean space [F], see also Sect. 19.3 in [H2], as explained in [BS], see also [A1]. Callias' index theorem was generalized by N. Anghel [A2] to Dirac operators coupled to the trivial Hermitian vector bundle over manifolds with warped cylindrical ends, using the relative index theory of [GL]. In this paper we generalize this index theorem to Dirac operators on arbitrary complete oriented spin-manifolds coupled to arbitrary Hermitian vector bundles.

Let $\lambda(x)$ denote the smallest of the absolute values of the eigenvalues of $\Phi(x)$. Let $\hat{A}$ denote the $\hat{A}$-genus. 
Theorem 1. If there exists a compact region $X_{0}$ in $X$, whose boundary is a smooth hypersurface $Y_{0}=\partial X_{0}$, and a constant $\lambda_{0}>0$, such that $\lambda(x) \geqq \lambda_{0}$ for $x \in X-X_{0}$, and $\left|\nabla_{A} \Phi(x)\right| \rightarrow 0$ as $x \rightarrow \infty$, then $D$ and $D^{*}$ have finite dimensional $L^{2}$-kernels, and

$$
\operatorname{dim} L^{2}-\operatorname{ker} D-\operatorname{dim} L^{2}-\operatorname{ker} D^{*}=-\int_{Y_{O}} \hat{A}\left(Y_{0}\right) \wedge \operatorname{ch}\left(V^{+}\right)=\int_{Y_{O}} \hat{A}\left(Y_{0}\right) \wedge \operatorname{ch}\left(V^{-}\right)
$$

where $V^{+}$denotes the span of the positive eigenvectors of $\Phi$, and $V^{-}$denotes the span of the negative eigenvectors of $\Phi$.

Note that the right-hand side is equal to minus the index of the chiral Dirac operator on $Y_{0}$ coupled to $V^{+}$. Sign conventions are discussed at the beginning of Sect. 2.

In Sect. 2 we prove a cutting and gluing lemma, Proposition 2.3. This lemma states that if we cut the manifold $X$ along a codimension one submanifold, and impose the right boundary conditions, then the index does not change. In Sect. 3 we use this cutting and gluing lemma to prove Theorem 1.

The condition $\left|\nabla_{A} \Phi(x)\right| \rightarrow 0$ can be weakened. Let $\pi^{+}: V \rightarrow V$ denote orthogonal projection onto $V^{+}$. It suffices that $\left|\nabla_{A} \pi^{+}\right|<\varepsilon_{n} \lambda_{0}$ on the complement of a compact subset, that could be larger than $X_{0}$, see Sect. 2 . The constant $\varepsilon_{n}$ depends on $n$ only.

The index can also be interpreted as a Fredholm index, as explained in Sect. 2. In particular, if $\Phi$, the curvature of the connection $A$, and the scalar curvature of $X$ are bounded, then $D$ is a Fredholm operator $L^{2,1}(X, S \otimes V) \rightarrow L^{2}(X, S \otimes V)$, with index as above.

In $[\mathrm{R}]$ we use this index theorem to compute the index of the anti-selfduality complex coupled to a singular Yang-Mills connection.

\section{A Cutting and Gluing Lemma}

Let $X$ be a complete odd-dimensional smooth oriented spin-manifold, with complex spinor bundle $S$, and Clifford multiplication $\gamma: \operatorname{Cliff}\left(T^{*} X\right) \otimes S \rightarrow S$. In this section, we allow $X$ to have compact boundary, $Y=\partial X$. We also allow $X$ to be non-compact, as long as it is complete. We orient the boundary of $X$ as follows: if $e_{1}, \ldots, e_{2 n}$ is an orthonormal frame on $Y$ and $v$ is the outward unit normal, then $v, e_{1}, \ldots, e_{2 n}$ is an orthonormal frame on $X$.

We use the normalization $e_{i}^{2}=-1$. Let $\omega_{X}=i^{n+1} e_{1} \ldots e_{2 n+1}$, where $e_{1}, \ldots, e_{2 n+1}$ is an orthonormal frame on $X$. Then $\omega_{X}$ is a global section of Cliff $\left(T^{*} X\right), \omega_{X}^{2}=1$ and $\omega_{X}$ lies in the center of Cliff $\left(T^{*} X\right)$. We use the normalization $\gamma\left(\omega_{X}\right)=1$.

The boundary $Y$ is also a spin-manifold, and its spinor bundle and Clifford multiplication is the restriction of the spinor bundle $S$ and Clifford multiplication $\gamma$ on $X$. Now, $Y$ is even-dimensional, so its spinor bundle splits into a positive and a negative spinor bundle,

$$
\left.S\right|_{Y}=S_{+} \oplus S_{-}
$$

Let $\omega_{Y}=i^{n} e_{1} \ldots e_{2 n}$, where $e_{i}, \ldots, e_{2 n}$ is an orthonormal frame on $Y$. Then $\omega_{Y}$ is a global section of $\operatorname{Cliff}\left(T^{*} Y\right)$, and $\omega_{Y}^{2}=1$. The positive and negative spinor bundles are the +1 and -1 eigenspaces of $\gamma\left(\omega_{Y}\right)$. By our orientation conventions, $\left.\omega_{X}\right|_{Y}=i v \omega_{Y}$, and

$$
1=\gamma\left(\left.\omega_{X}\right|_{Y}\right)=i \gamma(v) \gamma\left(\omega_{Y}\right)
$$


Hence the positive and negative spinor bundles are the $-i$ and $+i$ eigenspaces of $\gamma(v)$,

$$
\left\{\begin{array}{l}
\gamma(v) s_{+}=-i s_{+} \\
\gamma(v) s_{-}=i s_{-}
\end{array}\right.
$$

Let $D=\oiint_{A}+i 1 \otimes \Phi$ be an operator as in Theorem 1, except that we now allow $X$ to have a compact boundary $Y$. For simplicity, we write $D=\not \partial+i \Phi$. It is an elliptic operator. It admits well known elliptic (coercive, Lopatinski type) boundary conditions, see for instance [S]. One such boundary condition is $s_{+}=0$, where $\left.s\right|_{Y}=s_{+}+s_{-}$is the decomposition of $s$ according to the splitting $\left.(S \otimes V)\right|_{Y}=\left(S_{+} \otimes V\right) \oplus\left(S_{-} \otimes V\right)$. The adjoint boundary condition is $s_{-}=0$.

To verify that the boundary condition $s_{+}=0$ is elliptic, freeze the coefficients of the operators at a point on the boundary, to get the boundary value problem

$$
\begin{cases}\not s_{s}=0 & \text { on }(-\infty, 0] \times \mathbb{R}^{2 n} \\ s_{+}=0 & \text { on }\{0\} \times \mathbb{R}^{2 n}\end{cases}
$$

One has to check that there are no bounded non-zero solutions of the form

$$
s(t, x)=s(t) \exp (i \xi \cdot x)
$$

with $\xi \neq 0$. This leads to a system of ordinary differential equations,

$$
\left\{\begin{array}{l}
\gamma(v) \partial_{t} s(t)+i \gamma(\xi) s(t)=0 \text { for } t \leqq 0 \\
s^{+}(0)=0
\end{array}\right.
$$

where $v$ denotes the positive unit vector field on $(-\infty, 0]$. Extend the splitting into positive and negative spinors on $\{0\} \times \mathbb{R}^{2 n}$ to $[0, \infty) \times \mathbb{R}^{2 n}$. By (2.1), the system can then be written

$$
\begin{cases}\partial_{t} s_{+}(t)-\gamma(\xi) s_{-}(t)=0 & \text { for } t \leqq 0 \\ \partial_{t} s_{+}(t)+\gamma(\xi) s_{-}(t)=0 & \text { for } t \leqq 0 \\ s_{+}(0)=0 . & \end{cases}
$$

It follows that

$$
\partial_{t}^{2} s_{+}(t)=-\gamma^{2}(\xi) s_{+}(t)=|\xi|^{2} s_{+}(t) .
$$

If $s_{+}(0)=0$ and $s_{+}(t)$ is bounded, then $s_{+}(t)=0$. By the first equation, $\gamma(\xi) s_{-}(t)=0$, so $s_{-}(t)=0$, and $s(t)=0$. We conclude that $s_{+}=0$ is an elliptic boundary condition for $D$. See for instance Sect. 10 in [H1] for more details on elliptic boundary value problems.

One can also form twisted boundary conditions. Let

$$
\left.V\right|_{Y}=V^{+} \oplus V^{-}
$$

be any splitting of $\left.V\right|_{Y}$, not necessarily related to $\Phi$. Then we can decompose $s$ as $s=s^{+}+s^{-}$on $Y$ according to the splitting $\left.(S \otimes V)\right|_{Y}=\left(S \otimes V^{+}\right) \oplus\left(S \otimes V^{-}\right)$. We can also decompose $s$ as $s=s_{+}^{+}+s_{+}^{-}+s_{-}^{+}+s_{-}^{-}$according to the splitting Then

$$
\left.(S \otimes V)\right|_{Y}=\left(S_{+} \otimes V^{+}\right) \oplus\left(S_{+} \otimes V^{-}\right) \oplus\left(S_{-} \otimes V^{+}\right) \oplus\left(S_{-} \otimes V^{-}\right) .
$$

$$
B s=s_{+}^{+}+s_{-}^{-}=0 \quad \text { on } Y
$$


is an elliptic boundary condition for $D$. The adjoint boundary condition is

$$
B^{*} s=s_{\mp}+s_{-}^{+}=0 \text { on } Y .
$$

Sometimes we write $B_{Y}$ or even $B_{X, Y}$ for $B$.

We need function spaces where $D$, and more generally, if $\partial X=Y \neq \emptyset, D \oplus B$, is Fredholm. Let $\eta$ be a smooth compactly supported cut-off function on $X$ that is equal to 1 near $Y$. Then we define $L_{D}^{2,1}(X, S \otimes V)$ as the completion of $C_{0}^{\infty}(X, S \otimes V)$ with respect to the norm

$$
\|\eta \nabla s\|_{L^{2}(X)}+\|(1-\eta) D s\|_{L^{2}(X)}+\|s\|_{L^{2}(X)} .
$$

It follows from standard elliptic theory that this norm is equivalent to the norm

$$
\|D S\|_{L^{2}(X)}+\|B S\|_{L^{2,1 / 2}(Y)}+\|s\|_{L^{2}(X)} .
$$

(It is unavoidable to introduce Sobolev spaces with fractional derivatives. Functions in $L^{2,1}$ have boundary values in $L^{2,1 / 2}$.)

It follows from the Bochner-Weitzenböck formula,

$$
\not^{2} s=\nabla^{*} \nabla s+\sum_{i, j} F_{i j} \gamma\left(e_{i}\right) \gamma\left(e_{j}\right) s+\frac{1}{4} R s,
$$

that if $\Phi$, the curvature $F$ of the connection $A$, and the scalar curvature $R$ of $X$ are bounded, then $L_{D}^{2,1}(X, S \otimes V)=L^{2,1}(X, S \otimes V)$.

Lemma 2.1. There exists a constant $\varepsilon_{n}>0$ with the following significance. If there exists $\lambda_{0}>0$ such that $\lambda(x) \geqq \lambda_{0}$ and $\left|\nabla_{A} \pi^{+}(x)\right| \leqq \varepsilon_{n} \lambda_{0}$ for all $x \in X$, and the boundary operator $B$ is given by splitting $V=V^{+} \oplus V^{-}$into positive and negative eigenspaces of $\Phi$, then $D \oplus B$ defines an invertible operator

$$
L_{D}^{2,1}(X, S \otimes V) \rightarrow L^{2}(X, S \otimes V) \oplus L^{2,1 / 2}\left(Y,\left(S_{+} \otimes V^{+}\right) \oplus\left(S_{-} \otimes V^{-}\right)\right) .
$$

Proof. Let $s \in L_{D}^{2,1}(X)$. Recall that $\not=\sum_{i} \gamma\left(e_{i}\right) \nabla_{i}$. Hence, by integration by parts and (2.1),

$$
\begin{aligned}
\int_{X} \not \partial s \cdot s d \mathrm{vol} & =\int_{X} s \cdot \not \partial s d \mathrm{vol}+\int_{Y} \gamma(v) s \cdot s d \mathrm{vol} \\
& =\int_{X} \overline{\not \partial s} \cdot s d \mathrm{vol}-\int_{Y} i\left(\left|s_{+}\right|^{2}-\left|s_{-}\right|^{2}\right) d \mathrm{vol} .
\end{aligned}
$$

(The integration by parts can be justified by multiplying by a sequence cut-off functions that exhaust $X$.) Take the imaginary part of this identity, to get

Hence

$$
\operatorname{Im} \int_{X} \not \partial s \cdot s d \mathrm{vol}=-\frac{1}{2} \int_{Y}\left(\left|s_{+}\right|^{2}-\left|s_{-}\right|^{2}\right) d \mathrm{vol} .
$$

$$
\operatorname{Im} \int_{X} D s \cdot s d \mathrm{vol}=\int_{X} \Phi_{S} \cdot s d \mathrm{vol}-\frac{1}{2} \int_{Y}\left(\left|s_{+}\right|^{2}-\left|s_{-}\right|^{2}\right) d \mathrm{vol} .
$$

Substitute $s^{+}$for $s$, to get

$$
\lambda_{0} \int_{X}\left|s^{+}\right|^{2} d \mathrm{vol} \leqq \int_{X} \Phi s^{+} \cdot s^{+} d \mathrm{vol} \leqq \operatorname{Im} \int_{X} D s^{+} \cdot s^{+} d \mathrm{vol}+\frac{1}{2} \int_{Y}\left|s_{+}^{+}\right|^{2} d \mathrm{vol} .
$$


Similarly,

$$
\lambda_{0} \int_{X}\left|s^{-}\right|^{2} d \mathrm{vol} \leqq-\int_{X} \Phi s^{-} \cdot s^{-} d \mathrm{vol} \leqq-\operatorname{Im} \int_{X} D s^{-} \cdot s^{-} d \mathrm{vol}+\frac{1}{2} \int_{Y}\left|s_{-}^{-}\right|^{2} d \mathrm{vol} .
$$

It follows that

$$
\begin{aligned}
\lambda_{0} \int_{X}|s|^{2} d \mathrm{vol} \leqq & \operatorname{Im} \int_{X}\left(D s^{+} \cdot s^{+}-D s^{-} \cdot s^{-}\right) d \mathrm{vol}+\frac{1}{2} \int_{Y}|B s|^{2} d \mathrm{vol} \\
= & \operatorname{Im} \int_{X} D s \cdot\left(s^{+}-s^{-}\right) d \mathrm{vol}+\frac{1}{2} \int_{Y}|B s|^{2} d \mathrm{vol} \\
& +\int_{X}\left(D s^{+} \cdot s^{-}-D s^{-} \cdot s^{+}\right) d \mathrm{vol}
\end{aligned}
$$

Now,

so

$$
\begin{aligned}
D s^{+} \cdot s^{-} & =\not s^{+} \cdot s^{-}+\Phi s^{+} \cdot s^{-} \\
& =\sum_{i} \gamma\left(e_{i}\right) \nabla_{i}\left(\pi^{+} s^{+}\right) \cdot s^{-}=\sum_{i} \gamma\left(e_{i}\right)\left(\nabla_{i} \pi^{+}\right) s^{+} \cdot s^{-},
\end{aligned}
$$

$$
\left|D s^{+} \cdot s^{-}\right| \leqq c_{n}\left|\nabla \pi^{+}\right|\left|s^{+}\right|\left|s^{-}\right| \leqq c_{n} \varepsilon_{n} \lambda_{0}|s|^{2} .
$$

The same bound applies to $D s^{-} \cdot s^{+}$. Hence, if we choose $\varepsilon_{n}=\left(4 c_{n}\right)^{-1}$, then

$$
\lambda_{0} \int_{X}|s|^{2} d \mathrm{vol} \leqq 2 \operatorname{Im} \int_{X} D s \cdot\left(s^{+}-s^{-}\right) d \mathrm{vol}+\int_{Y}|B s|^{2} d \mathrm{vol} .
$$

By Cauchy's inequality,

$$
\|s\|_{L^{2}(X)} \leqq 4 \lambda_{0}^{-2}\|D S\|_{L^{2}(X)}+2 \lambda_{0}^{-1}\|B S\|_{L^{2}(Y)} .
$$

Hence

$$
\|s\|_{L_{D}^{2,1}(X)} \leqq\left(4 \lambda_{0}^{-2}+1\right)\|D s\|_{L^{2}(X)}+\left(2 \lambda_{0}^{-1}+1\right)\|B S\|_{L^{2,1 / 2}(Y)} .
$$

This estimate shows that $D \oplus B$ has trivial kernel and closed range.

By integration by parts, the orthogonal complement of the range of $D: L_{D}^{2,1}(X) \cap \operatorname{ker} B \rightarrow L^{2}(X)$ is the $L^{2}$-kernel of $D^{*} \oplus B^{*}$. The same way as above, one shows that the $L^{2}$-kernel of $D^{*} \oplus B^{*}$ is trivial. Hence $D: L_{D}^{2,1}(X) \cap \operatorname{ker} B$ $\rightarrow L^{2}(X)$ has dense range. Hence $D \oplus B: L_{D}^{2,1}(X) \rightarrow L^{2}(X) \oplus L^{2,1 / 2}(Y)$ has dense range. Hence $D \oplus B$ is invertible.

We can use Lemma 2.1 to prove a more general Fredholm result. Let $\varepsilon_{n}$ be as in Lemma 2.1.

Lemma 2.2. If there exists a compact subset $X_{0}$ of $X$ and a constant $\lambda_{0}>0$, such that $\lambda(x) \geqq \lambda_{0}$ and $\left|\nabla_{A} \pi^{+}(x)\right| \leqq \varepsilon_{n} \lambda_{0}$ for all $x \in X-X_{0}$, then the operator

$D \oplus B: L_{D}^{2,1}(X, S \otimes V) \rightarrow L^{2}(X, S \otimes V) \oplus L^{2,1 / 2}\left(Y,\left(S_{+} \otimes V^{+}\right) \oplus\left(S_{-} \otimes V^{-}\right)\right)$

is Fredholm. The Fredholm index of $D \oplus B$ is equal to the $L^{2}$-index

$$
\operatorname{dim} L^{2}-\operatorname{ker}(D \oplus B)-\operatorname{dim} L^{2}-\operatorname{ker}\left(D^{*} \oplus B^{*}\right) .
$$

Here $B$ can be given by any splitting $\left.V\right|_{\partial X}=V^{+} \oplus V^{-}$. 
Proof. After enlarging $X_{0}$, we may assume that it is bounded by a smooth hypersurface, and that $\partial X \subset \partial X_{0}$. Let $X_{\infty}=X-X_{0}$. Enlarge $X_{\infty}$ slightly, so that $\left\{X_{0}, X_{\infty}\right\}$ is a good cover of $X$.

The splitting $V=V^{+} \oplus V^{-}$is only defined on $\partial X$. We extend the splitting to $\partial X_{0}-\partial X$ in some arbitrary way. We extend the splitting to $\partial X_{\infty}$ by letting $V^{+}$ and $V^{-}$be the span of the positive and negative eigenspaces of $\Phi$ respectively.

By standard elliptic theory $D_{X_{0}} \oplus B_{\partial X_{0}}$ has a parametrix $P_{0}$. By Lemma 2.1, $D_{X_{\infty}} \oplus B_{\partial X_{\infty}}$ has an inverse $P_{\infty}$. It is then standard to construct a parametrix $P$ for $D \oplus B$ using $P_{0}, P_{\infty}$, and a partition of unity subordinate to the cover $\left\{X_{0}, X_{\infty}\right\}$.

It is clear that the $L_{D}^{2,1}$-kernel of $D \oplus B$ is equal to the $L^{2}$-kernel of $D \oplus B$. The cokernel of $D \oplus B$ can be identified with the cokernel of $D: L_{D}^{2,1}(X) \cap \operatorname{ker} B$ $\rightarrow L^{2}(X)$. Integration by parts shows that the orthogonal complement of the image of this operator is the $L^{2}$-kernel of $D^{*} \oplus B^{*}$.

Now, let $X$ be a manifold as above, that has been obtained by gluing two complete spin-manifolds $X_{1}$ and $X_{2}$, with boundaries $\partial X_{1}=Y \cup Y_{1}$ and $\partial X_{2}=\bar{Y}$ $\cup Y_{2}$, along $Y$. (Here $\bar{Y}$ denotes $Y$ with the opposite orientation.) We write $X=X_{1} \cup_{Y} X_{2}$. Then $\partial X=Y_{1} \cup Y_{2}$. We write $D_{X}$ for the operator $D$ on $X$, and $D_{X_{i}}$ for its restriction to $X_{i}$.

\section{Proposition 2.3.}

$$
\text { index }\left(D_{X} \oplus B_{X, Y_{1} \cup Y_{2}}\right)=\operatorname{index}\left(D_{X_{1}} \oplus B_{X_{1}, Y \cup Y_{1}}\right)+\operatorname{index}\left(D_{X_{2}} \oplus B_{X_{2}, \bar{Y} \cup Y_{2}}\right) .
$$

Here $B$ can be given by any splitting $\left.V\right|_{Y \cup Y_{1} \cup Y_{2}}=V^{+} \oplus V^{-}$.

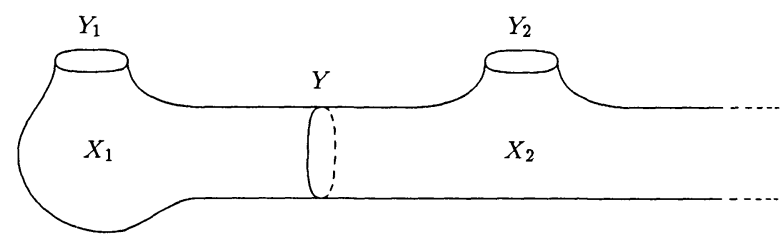

Proof. For simplicity, we assume that $Y_{1}=Y_{2}=\emptyset$. This is only to simplify the notation; the general case is handled the same way.

By Lemma 2.1, $D_{X_{1}} \oplus D_{X_{2}} \oplus B_{X_{1}, Y} \oplus B_{X_{2}, \bar{Y}}$ is a Fredholm operator

$$
\begin{aligned}
L_{D}^{2,1}\left(X_{1}, S \otimes V\right) \oplus L_{D}^{2,1}\left(X_{2}, S \otimes V\right) \rightarrow & L^{2}\left(X_{1}, S \otimes V\right) \oplus L^{2}\left(X_{2}, S \otimes V\right) \\
& \oplus L^{2,1 / 2}\left(Y,\left(S_{+} \otimes V^{+}\right) \oplus\left(S_{-} \otimes V^{-}\right)\right) \\
& \oplus L^{2,1 / 2}\left(\bar{Y},\left(S_{+} \otimes V^{+}\right) \oplus\left(S_{-} \otimes V^{-}\right)\right) .
\end{aligned}
$$

If we identify $Y$ and $\bar{Y}$, then

$$
L^{2}\left(\bar{Y},\left(S_{+} \otimes V^{+}\right) \oplus\left(S_{-} \otimes V^{-}\right)\right)=L^{2}\left(Y,\left(S_{-} \otimes V^{+}\right) \oplus\left(S_{+} \otimes V^{-}\right)\right) .
$$

Hence the boundary operators $B_{X_{1}, Y}$ and $B_{X_{2}, \bar{Y}}$ can be subtracted to form one operator

$$
\begin{gathered}
B^{(0)}: L_{D}^{2,1}\left(X_{1}, S \otimes V\right) \oplus L_{D}^{2,1}\left(X_{2}, S \otimes V\right) \rightarrow L^{2,1 / 2}(Y, S \otimes V) \\
B^{(0)}=B_{X_{1}, Y}-B_{X_{2}, \bar{Y}} .
\end{gathered}
$$


More generally, we define

$$
\begin{gathered}
B^{(\theta)}: L_{D}^{2,1}\left(X_{1}, S \otimes V\right) \oplus L_{D}^{2,1}\left(X_{2}, S \otimes V\right) \rightarrow L^{2,1 / 2}(Y, S \otimes V) \\
B^{(\theta)}=B_{X_{1}, Y}-B_{X_{2}, \bar{Y}}+\theta\left(B_{X_{1}, Y}^{*}-B_{X_{2}, \bar{Y}}^{*}\right) .
\end{gathered}
$$

The proposition follows from the following three facts.

a. The operators $D_{X_{1}} \oplus D_{X_{2}} \oplus B^{(\theta)}$ form a continuous (in the norm topology) family of Fredholm operators

$$
\begin{aligned}
& L_{D}^{2,1}\left(X_{1}, S \otimes V\right) \oplus L_{D}^{2,1}\left(X_{2}, S \otimes V\right) \\
& \quad \rightarrow L^{2}\left(X_{1}, S \otimes V\right) \oplus L^{2}\left(X_{2}, S \otimes V\right) \oplus L^{2,1 / 2}(Y, S \otimes V),
\end{aligned}
$$

parametrized by $\theta \in \mathbb{R}$.

b. index $\left(D_{X_{1}} \oplus D_{X_{2}} \oplus B^{(0)}\right)=\operatorname{index}\left(D_{X_{1}} \oplus B_{X_{1}, Y}\right)+\operatorname{index}\left(D_{X_{2}} \oplus B_{X_{2}, \bar{Y}}\right)$.

c. $\operatorname{index}\left(D_{X_{1}} \oplus D_{X_{2}} \oplus B^{(1)}\right)=\operatorname{index}\left(D_{X}\right)$.

Proof of b. Essentially, $B^{(0)}=B_{X_{1}, Y} \oplus B_{X_{2}, \bar{Y}}$.

Proof of c. Let $s_{1} \oplus s_{2} \in L_{D}^{2,1}\left(X_{1}\right) \oplus L_{D}^{2,1}\left(X_{2}\right)$. Then $B^{(1)}\left(s_{1} \oplus s_{2}\right)=\left.s_{1}\right|_{Y}-\left.s_{2}\right|_{\bar{Y}}$. The sections $s_{1}$ and $s_{2}$ can be glued to form a section $s \in L_{D}^{2,1}(X)$ if and only if $B^{(1)}\left(s_{1} \oplus s_{2}\right)=0$. Hence we have a commutative diagram with exact rows,

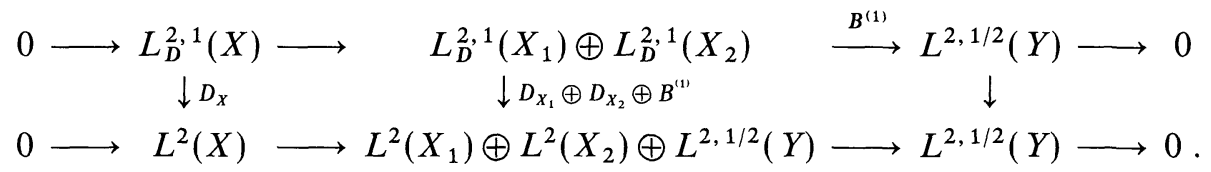

The claim follows from this diagram.

Proof of a. This is a boundary value problem where the boundary conditions mix different boundary components. Such boundary value problems are known as transmission problems. By identifying a neighborhood of $Y$ in $X_{1}$ with a neighborhood of $\bar{Y}$ in $\bar{X}_{2}$ one gets a elliptic boundary condition in the usual sense. Thus the ellipticity (coerciveness, Lopatinski) condition is still meaningful, and ellipticity implies the existence of local parametrices near the boundary. See for instance [H1] p. 274.

Freeze the coefficients at a point on $Y$, to get the transmission problem

$$
\begin{cases}\not s_{1}=0 & \text { on }(-\infty, 0] \times \mathbb{R}^{2 n} \\ \not s_{2}=0 & \text { on }[0, \infty) \times \mathbb{R}^{2 n} \\ \left(s_{1}\right)_{+}-\theta \cdot\left(s_{2}\right)_{+}=0 & \text { on }\{0\} \times \mathbb{R}^{2 n} \\ \left(s_{2}\right)_{-}-\theta \cdot\left(s_{1}\right)_{-}=0 & \text { on }\{0\} \times \mathbb{R}^{2 n} .\end{cases}
$$

To verify ellipticity, one has to check that there are no bounded non-zero solutions of the form

$$
\begin{cases}s_{1}(t, x)=s_{1}(t) \exp (i \xi \cdot x) & \text { for } t \leqq 0 \\ s_{2}(t, x)=s_{2}(t) \exp (i \xi \cdot x) & \text { for } t \geqq 0\end{cases}
$$


with $\xi \neq 0$. This leads to a system of ordinary differential equations,

$$
\begin{cases}\gamma(v) \partial_{t} s_{1}(t)+i \gamma(\xi) s_{1}(t)=0 & \text { for } t \leqq 0 \\ \gamma(v) \partial_{t} s_{2}(t)+i \gamma(\xi) s_{2}(t)=0 & \text { for } t \geqq 0 \\ s_{1}(0)_{+}-\theta s_{2}(0)_{+}=0 & \\ s_{2}(0)_{-}-\theta s_{1}(0)_{-}=0 & \end{cases}
$$

It is not hard to check that there are no bounded non-zero solutions with $\xi \neq 0$.

Hence there exists a parametrix on a neighborhood of $Y$. Then one can argue as in the proof of Lemma 2.2 to show that the operator is Fredholm.

Remark 2.4. We have only considered the boundary operator $B$. However, Lemma 2.2 and Proposition 2.3 apply mutatis mutandis to $B^{*}$, for $B^{*}$ is $\operatorname{simply} B$ with $V^{+}$ and $V^{-}$switched.

\section{Proof of the Index Theorem}

Proof of Theorem 1. By Lemma 2.2, the operator

$$
D: L_{D}^{2,1}(X, S \otimes V) \rightarrow L^{2}(X, S \otimes V)
$$

is Fredholm. Let $X_{\infty}=X-X_{0}$. Then $\partial X_{\infty}=\bar{Y}_{0}$. By Proposition 2.3,

$$
\text { index } D \equiv \operatorname{index} D_{X}=\operatorname{index}\left(D_{X_{0}} \oplus B_{Y_{0}}\right)+\operatorname{index}\left(D_{X_{\infty}} \oplus B_{\bar{Y}_{0}}\right) \text {. }
$$

Now,

$$
\operatorname{index}\left(D_{X_{0}} \oplus B_{Y_{0}}\right)=\operatorname{index}\left(\not_{X_{0}} \oplus B_{Y_{0}}\right)
$$

for a lower order term $\Phi$ does not affect the index on a compact manifold $X_{0}$. Also, $\left|\nabla_{A} \pi^{+}\right| \leqq \lambda^{-1}\left|\nabla_{A} \Phi\right| \rightarrow 0$ on the ends of $X$. Hence we can deform the connection $A$ smoothly on a compact set, so that $\left|\nabla_{A} \pi^{+}\right| \leqq \varepsilon_{n} \lambda_{0}$ on $X-X_{0}$. Then, by Lemma 2.1,

Hence

$$
\operatorname{index}\left(D_{X_{\infty}} \oplus B_{\bar{Y}_{0}}\right)=0 \text {. }
$$

$$
\text { index } D=\operatorname{index}\left(\not_{X_{0}} \oplus B_{Y_{0}}\right) \text {. }
$$

Thus the index problem on $X$ with $L^{2}$-boundary conditions is equivalent to an index problem on $X_{0}$ with elliptic boundary conditions. In other words, elliptic boundary conditions play the same role in Callias' index theorem as spectral boundary conditions do in the Atiyah-Patodi-Singer index theorem [APS].

There are many ways to compute the index of $D_{X_{0}} \oplus B_{Y_{0}}$. One could use the Atiyah-Bott index theorem [AB] for elliptic boundary value problems. One can also argue as follows. By Lemma 2.2,

$$
\operatorname{index}\left(\not_{X_{0}} \oplus B_{Y_{0}}\right)+\operatorname{index}\left(\not_{X_{0}} \oplus B_{Y_{0}}^{*}\right)=0 \text {. }
$$

On the other hand, it follows from the Agranovich-Dynin difference theorem, [AD] Theorem 2, that

$$
\operatorname{index}\left(\not_{X_{0}} \oplus B_{Y_{0}}\right)-\operatorname{index}\left(\not \not_{X_{0}} \oplus B_{Y_{0}}^{*}\right)=\operatorname{index}\left(\not \not_{Y_{0}}\right)_{-}^{+}+\operatorname{index}\left(\not_{Y_{0}}\right)_{+}^{-},
$$


where

$$
\begin{aligned}
& \left(\partial_{Y_{0}}\right)_{+}^{ \pm}: S_{+} \otimes V^{ \pm} \rightarrow S_{-} \otimes V^{ \pm}, \\
& \left(\partial_{Y_{0}}\right)^{ \pm}: S_{-} \otimes V^{ \pm} \rightarrow S_{+} \otimes V^{ \pm}
\end{aligned}
$$

denote the chiral Dirac operators on $Y_{0}$ coupled to $V^{ \pm}$.

This can also be seen as follows. We can assume that the metric on $X_{0}$ is a product metric on a collar $[-\delta, 0] \times Y_{0}$ of $Y_{0}$. We can assume that the bundle has a fixed product structure on $[-\delta, 0] \times Y_{0}$, that the connection $A$ is the pullback of a connection on $Y_{0}$, and that this connection is reducible with respect to the splitting $V=V^{+} \oplus V^{-}$. Form a smooth manifold $X_{0} \cup_{Y_{0}}\left([0,1] \times Y_{0}\right)$ by gluing $Y_{0}=\partial X_{0}$ to $\{0\} \times \bar{Y}_{0}$. Note that

$$
\partial\left([0,1] \times Y_{0}\right)=\left(\{0\} \times \bar{Y}_{0}\right) \cup\left(\{1\} \times Y_{0}\right) .
$$

The bundle $V$ and the connection $A$ extend naturally to $X_{0} \cup_{Y_{0}}\left([0,1] \times Y_{0}\right)$.

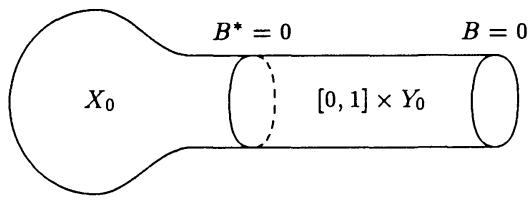

We can continuously deform $X_{0}$ to $X_{0} \cup_{Y_{0}}\left([0,1] \times Y_{0}\right)$ along with the operators, so

$$
\operatorname{index}\left(\not_{X_{0}} \oplus B_{Y_{0}}\right)=\text { index }\left(\not_{X_{0} \cup_{Y_{0}}\left([0,1] \times Y_{0}\right)} \oplus B_{\{1\} \times Y_{0}}\right) \text {. }
$$

By Proposition 2.3 and Remark 2.4,

$$
\begin{aligned}
& \operatorname{index}\left(\not_{X_{0} \cup_{Y_{0}}\left([0,1] \times Y_{0}\right)} \oplus B_{\{1\} \times Y_{0}}\right) \\
& \quad=\operatorname{index}\left(\not \not_{X_{0}} \oplus B_{Y_{0}}^{*}\right)+\operatorname{index}\left(\not \not_{[0,1] \times X_{0}} \oplus B_{\{0\} \times \bar{Y}_{0}}^{*} \oplus B_{\{1\} \times Y_{0}}\right) .
\end{aligned}
$$

Hence,

$$
\operatorname{index}\left(\not \not_{X_{0}} \oplus B_{Y_{0}}\right)-\operatorname{index}\left(\not \not_{X_{0}} \oplus B_{Y_{0}}^{*}\right)=\operatorname{index}\left(\not_{[0,1] \times Y_{0}} \oplus B_{\{0\} \times \bar{Y}_{0}}^{*} \oplus B_{\{1\} \times Y_{0}}\right) \text {. }
$$

We will now show that the kernel and cokernel of the operator on the right-hand side is given by harmonic spinors on $Y_{0}$. The splittings $S=S_{+} \oplus S_{-}$and $V=V^{+} \oplus V^{-}$on $Y_{0}$ extend naturally to $[0,1] \times Y_{0}$. Note that with this notation, $S_{+}$is the negative spinor bundle on $\{0\} \times \bar{Y}_{0}$ and vice versa. Separate variables on $[0,1] \times Y_{0}$. Let $t$ be the $[0,1]$-coordinate. Let $v$ denote the unit vector field in the $t$-direction; it is the natural extension of the outward unit normal vector field $v$ on $\{1\} \times Y_{0}$. Then $\not_{[0,1] \times Y_{0}}=\gamma(v) \partial_{t}+\not_{Y_{0}}$. By $(2.1)$, for $s=s_{+}^{+} \oplus s_{+}^{-} \oplus s_{-}^{+} \oplus s_{-}^{+}$, we have

$$
\begin{aligned}
\not \partial s= & \left(\not \partial_{Y_{0}} s_{-}^{+}-i \partial_{t} s_{+}^{+}\right) \oplus\left(\not \partial_{Y_{0}} s_{-}^{-}-i \partial_{t} s_{+}^{-}\right) \\
& \oplus\left(\not_{Y_{0}} s_{+}^{+}+i \partial_{t} s_{-}^{+}\right) \oplus\left(\not_{Y_{0}} s_{+}^{-}+i \partial_{t} s_{-}^{-}\right) .
\end{aligned}
$$

The boundary condition is tantamount to $s_{+}^{+}=s_{-}^{-}=0$ on $\partial\left([0,1] \times Y_{0}\right)$. (On $\{0\} \times \bar{Y}_{0}$ we have imposed a $B^{*}$-condition, but $S_{+}$is the negative spin bundle on $\{0\} \times \bar{Y}_{0}$ and vice versa.) Hence, if $s_{+}^{-}$is a harmonic $S_{+} \otimes V^{-}$-spinor on $Y_{0}$, then its pull back to $[0,1] \times Y_{0}$ is in the kernel. The same holds for harmonic $S_{-} \otimes V^{+}$spinors on $Y_{0}$. I claim that this gives the whole kernel. 
To see this, assume that $s$ is in the kernel. Then $\not \partial^{2} s_{+}^{+}=(\not 2)_{+}^{+}=0$ on $[0,1] \times Y_{0}$. Since $s_{+}^{+}=0$ on $\partial[0,1] \times Y_{0}$, we can integrate by parts to get

$$
\begin{aligned}
0 & =\int_{[0,1] \times Y_{0}}\left|\partial_{[0,1] \times Y_{0}} s_{+}^{+}\right|^{2} d \mathrm{vol}=\int_{[0,1] \times Y_{0}} s_{+}^{+} \cdot \not_{[0,1] \times Y_{0}}^{2} s_{+}^{+} d \mathrm{vol} \\
& =\int_{[0,1] \times Y_{0}} s_{+}^{+} \cdot\left(-\partial_{t}^{2}+\not \partial_{Y_{0}}^{2}\right) s_{+}^{+} d \mathrm{vol}=\int_{[0,1] \times Y_{0}}\left(\left|\partial_{t} s_{+}^{+}\right|^{2}+\left|\not \partial_{Y_{0}} s_{+}^{+}\right|^{2}\right) d \mathrm{vol} .
\end{aligned}
$$

Hence $\partial_{t} s_{+}^{+}=0$ on $[0,1] \times Y_{0}$. By the boundary condition, $s_{+}^{+}=0$ on $\partial\left([0,1] \times Y_{0}\right)$. Hence $s_{+}^{+}=0$ on $[0,1] \times Y_{0}$. Similarly $s_{-}^{-}=0$ on $[0,1] \times Y_{0}$. It then follows from (3.4) that $\not_{Y_{0}} s=0$ and $\partial_{t} s=0$, and the claim follows.

To summarize,

$$
\operatorname{ker}\left(\not_{[0,1] \times Y_{0}} \oplus B_{\{0\} \times \bar{Y}_{0}}^{*} \oplus B_{\{1\} \times Y_{0}}\right) \cong \operatorname{ker}\left(\not_{Y_{0}}\right)_{+}^{-} \oplus \operatorname{ker}\left(\not_{Y_{0}}\right)_{-}^{+} .
$$

Similarly,

$$
\operatorname{ker}\left(\not_{[0,1] \times Y_{0}} \oplus B_{\{0\} \times \bar{Y}_{0}} \oplus B_{\{1\} \times Y_{0}}^{*}\right) \cong \operatorname{ker}\left(\not_{Y_{0}}\right)_{+}^{+} \oplus \operatorname{ker}\left(\not_{Y_{0}}\right)_{-}^{-} .
$$

Hence,

$$
\begin{aligned}
\operatorname{index}( & \left.\not_{[0,1] \times Y_{0}} \oplus B_{[0,1] \times X_{0},\{0\} \times \bar{Y}_{0}}^{*} \oplus B_{[0,1] \times X_{0},\{1\} \times Y_{0}}\right) \\
= & \operatorname{dim} \operatorname{ker}\left(\not_{[0,1] \times Y_{0}} \oplus B_{\{0\} \times \bar{Y}_{0}}^{*} \oplus B_{\{1\} \times Y_{0}}\right) \\
& -\operatorname{dim} \operatorname{ker}\left(\not_{[0,1] \times Y_{0}} \oplus B_{\{0\} \times \bar{Y}_{0}} \oplus B_{\{1\} \times \bar{Y}_{0}}^{*}\right) \\
= & \operatorname{dim} \operatorname{ker}\left(\not_{Y_{0}}\right)_{+}^{-}+\operatorname{dim} \operatorname{ker}\left(\not_{Y_{0}}\right)_{-}^{+}-\operatorname{dim} \operatorname{ker}\left(\not_{Y_{0}}\right)_{+}^{+}-\operatorname{dim} \operatorname{ker}\left(\not_{Y_{0}}\right)_{-}^{-} \\
= & \operatorname{index}\left(\not_{Y_{0}}\right)_{+}^{-}+\operatorname{index}\left(\not_{Y_{0}}\right)_{-}^{+},
\end{aligned}
$$

and we have proven (3.3).

By the Atiyah-Singer index theorem,

$$
\left\{\begin{array}{l}
\operatorname{index}\left(\not \partial_{Y_{0}}\right)_{+}^{+}=-\operatorname{index}\left(\not_{Y_{0}}\right)_{-}^{+}=\int_{Y_{0}} \hat{A}\left(Y_{0}\right) \wedge \operatorname{ch}\left(V^{+}\right) \\
\operatorname{index}\left(\not \partial_{Y_{0}}\right)_{+}^{-}=-\operatorname{index}\left(\not \partial_{Y_{0}}\right)_{-}^{-}=\int_{Y_{0}}\left(\hat{A}\left(Y_{0}\right) \wedge \operatorname{ch}\left(V^{-}\right) .\right.
\end{array}\right.
$$

Finally, the forms $\hat{A}\left(Y_{0}\right)$ and $\operatorname{ch}(V)$ extend as closed forms across $X_{0}$. By Stokes' theorem

$$
\int_{Y_{0}} \hat{A}\left(Y_{0}\right) \wedge\left(\operatorname{ch}\left(V^{+}\right)+\operatorname{ch}\left(V^{-}\right)\right)=\int_{Y_{0}} \hat{A}\left(Y_{0}\right) \wedge \operatorname{ch}(V)=0 .
$$

The theorem now follows from (3.1)-(3.3), (3.5), and (3.6).

Acknowledgements. The author wishes to thank Dan Freed, Lars Hörmander, Rafe Mazzeo, Tom Mrowka and Karen Uhlenbeck for interesting discussions and valuable comments.

Note. Theorem 1 has been obtained independently by N. Anghel [A3].

\section{References}

[A1] Anghel, N.: Remark on Callias' index theorem. Rep. Math. Phys. 28, 1-6 (1988)

[A2] Anghel, N.: $L^{2}$-index formulae for perturbed Dirac operators. Commun. Math. Phys. 128, 77-97 (1990) 
[A3] Anghel, N.: The index of Callias-type operators. Preprint, University of North Texas

[AD] Agranovich, M.S , Dynin, A.S : General boundary value problems for elliptic systems in an $n$-dimensional domain. Dokl. Akad. Nauk SSSR Ser. Mat. 146, 511-514 (1962) [English transl.: Soviet Math. Dokl. 3, 1323-1327 (1962)

[AB] Atiyah, M F , Bott, R : The index theorem for manifolds with boundary. In: Differential Analysis (Bombay 1964). Oxford: Oxford University Press, 1964, pp. 175-186

[APS] Atiyah, M.F., Patodi, V.K, Singer, I.M.: Spectral asymmetry and Riemannian geometry I Math. Proc. Camb. Phil. Soc. 77, 43-69 (1975)

[BS] Bott, R., Seeley, R : Some remarks on the paper of Callias. Commun. Math. Phys. 62, 236-245 (1978)

[C] Callias, C.: Axial anomalies and index theorems on open spaces. Commun Math. Phys. 62, 213-234 (1978)

[F] Fedosov, B.V.: Analytic formulas for the index of elliptic operators. Trudy Mosk Mat. Obšč. 30, 159-241 (1974) [English transl.: Trans. Mosc. Math. Soc. 30, 159-240 (1974)

[GL] Gromov, M., Lawson, H.B.: Positive scalar curvature and the Dirac operator. Publ. Math IHES 58, 83-196 (1983)

[H1] Hörmander, L.: Linear Partial Differential Operators. Grundl. math. Wiss. vol. 116, Berlin, Heidelberg, New York: Springer 1964

[H2] Hörmander, L.: The Analysis of Linear Partial Differential Operators III. Grundl. math. Wiss vol 274, Berlin, Heidelberg, New York: Springer 1985

[R] Råde, J.: Singular Yang-Mills fields II. Global theory. Preprint, Stanford University 1993

[S] Singer, I.M.: The $\eta$-invariant and the index. In: S.-T. Yau (ed.) Mathematical Aspects of String Theory (San Diego 1986). Singapore: World Scientific, 1987, pp. 239-258

Communicated by G. Felder 
